\title{
An Efficient Machine Learning Regression Model for Rainfall Prediction
}

\author{
R. Usha Rani \\ Research Scholar, \\ Krishna University, \\ India.
}

\author{
T.K.Rama Krishna Rao \\ Principal, Department of IT, \\ AITAM college, \\ India.
}

\author{
R. Kiran Kumar Reddy \\ Department of CSE, \\ Krishna University, \\ India.
}

\begin{abstract}
Interfacing through the continuously rising amounts of data in technical, medical, scientific, engineering, industrial and monetary fields and their renovation to logical form for the human user is one of the main requirements. To quickly discover and analyze complex patterns and requirements, we need the efficient techniques and need to learn from new data will be necessary for information-intensive applications. One of the solutions for this is that classification and clustering of largely available data. To partially fulfill the industry requirement, in this paper we proposed a two-level approach for clustering large data set for rain fall data prediction with Self Organized Maps (SOM) and Support Vector Machine (SVM) with ID3. In this paper, a novel approach to clustering of the SOM and SVM with ID3 are considered. In particular, the use of hierarchical agglomerative clustering and partitioned clustering with ID3 are investigated. The twostage procedure first using SOM to produce the prototypes and later it considers the SVM with ID3, that are then clustered in the second stage is found to perform well when compared with direct clustering of the data and to reduce the computation time.
\end{abstract}

\section{Keywords}

SOM, SVM, ID3, Clustering, Classification, Data mining.

\section{INTRODUCTION}

Over the previous decade, academic and commercialized databases have been extending at exceptional rates. Capture advanced perception from such databases is hard, expansive and time-consuming if done manually. It is hopeless when data exceeds definite limits of size and complexity. For this reason, during the previous years the automated analysis and visualization of huge multi-dimensional datasets has been the center of attention on scientific research. The fundamental aim is to observe rules and relationships in the data, thereby gaining attain to invisible and potentially valuable knowledge [1-5]. Artificial Neural Networks are a hopeful part of this broad field. Motivated by advances in biomedical research, they shape a class of algorithms that goal to reproduce the neural structures of the brain. The Self-Organizing Map (SOM) is an absolutely popular neural network and actually one of the most desired unsupervised learning algorithms. Since its creation by Finnish Professor Teuvo Kohonen in the early 1980s, additional 4000 research artifacts have been published on the algorithm, its visualization and applications. The maps perfectly visualize natural sets and relationships in the data and have been effectively applied in a broad spectrum of research areas ranging from speech recognition to financial analysis [6-8].

In information visualization the Self-Organizing Map and SVM is an amazing tool. Even though mathematical analysis of the algorithm is still alive, its fundamental implementation is simple and the map allegory easy to understand. Moreover, the results are believable and the algorithm scales extraordinarily well. The process of data visualizing and understanding in many studies and applications SOM's and SVM's has confirm to be an excellent. In machine learning, SOM and SVM are supervised learning models with associated learning algorithms that examine data and recognize patterns, used for classification and regression analysis. Specified set of training examples, individually marked as propitious to one of two classifications, an SOM and SVM training algorithm frames a model that consigns new examples into one category or the other, manufacture it a non-probabilistic binary linear classifier [8-12]. In this paper, we especially, focus on Weather prediction with SOM and SVM with ID3, which are a ticklishness poll and a tough task for investigators. It contains skill in multiple disciplines $[1,2]$. The forecast of atmospheric parameters is crucial for various applications. Some of them include weather monitoring, drought detection, serious weather prediction, farming and production, planning in energy industry, aeronautical industry, communication, contamination dispersal etc [6].

The rest of the paper is organized as follows. Section II analyzes about existing mechanisms. Section III discusses about system structure. Section IV discusses the results of the proposed mechanism. Finally, Section V concludes the paper.

\section{RELATED WORK}

Data analysis underlies many computing applications, either in a design phase or as part of their on-line operations. Data analysis procedures can be dichotomized as either exploratory or confirmatory, based on the availability of appropriate models for the data source, but a key element in both types of procedures is the grouping, or classification of measurements based on either (i) goodness-of-fit to a postulated model, or (ii) natural groupings (clustering) revealed through analysis. Cluster analysis is the organization of a collection of patterns into clusters based on similarity [13-16]

\subsection{Clustering}

Data mining is the exploration and analysis of large quantities of data in order to discover valid, novel, potentially useful, and ultimately understandable patterns in data. Progressively robust computer hardware has made it feasible to examine and probe databases whose complexity, dimensionality and amount of data contravene the limits in which manual analysis is feasible [17-20]. The motive is to divulge patterns, relationships or regularities that grant us to gain new knowledge and intuition on the data.

Machine Learning supplies the technical basis for Data Mining and the SOM and SVM are one of the multiple algorithms used in this occasional. More accurately, these belong to the class of Neural Network algorithms. This is a group of algorithms based on analogies to the neural structures of the brain. The SOM in exclusive was motivated by an engrossing phenomenon: As physicians have find, some areas of brain tissue can be instructed according to an input 
signal basically, the SOM and SVM is a computer program simulating this ordering process.

\subsection{The Self-Organizing Map}

Investigative data mining use artificial neural networks proffers a substitute dimension to data mining, in specific techniques geared towards data clustering and classification. we use neural networks as a feasible data mining tool that can grant statistical intuition and models from large data-sets and how Self-Organizing Kohonen Maps, an unsupervised learning neural network paradigm, can be successfully used for data mining purposes, in exclusive for data clustering applications. We exhibit that high-dimensional data can be estimate to a lower dimension and that data can be clustered conjointly whilst preserving necessary information. In 1991 World Bank social economic indicators use Kohonen Map based clustering techniques for showing two-dimensional maps from multidimensional data sets, and also show clusters of similar data items. The SOM is exclusively acceptable for data survey because it has important visualization properties. It generates a group of prototype vectors representing the data set and fulfill a topology protect projection of the prototypes from the $\mathrm{d}$-dimensional input space onto a low-dimensional grid [4-5]. This ordered grid can be used as a convenient visualization surface for showing different features of the SOM (and thus of the data).

\section{Components of Self-Organizing Map}

SOM having two components first one is sample data and second one is weight vectors.

Sample data: The first part of SOM deals with the data as some examples as 3 dimensional data which are commonly used when experimenting with SOMs [13-24]. Here three dimensions represents colors these are red, blue, and green. The main aim of SOM is provide best visualization to ndimensional data.

Weights: Each weight vector contains two parts. The first part represents its data and second part represents natural location

Self-Organizing Map Applications: The SelfOrganization Map used several areas some of the application areas given below

Speech Recognition: The main aim of SOM in speech recognition is to develop objective indicators for increase quality of voice. In speech recognition use Teuvo Kohonen's pioneering work on Self-Organizing Maps.

Control Engineering: SOM is used as a look-up table in engineering applications. The Look-up table gives similar row in a given input pattern. By using learning phase of algorithm [20-24] we find best matching unit in a map.

Biomedical Sciences: Self-Organizing Maps have considerably contributed to the upcoming research area of Bioinformatics.

Financial Analysis: The SOM has been used by many writers including Kaski et al. to search financial statements of enterprises and to divulge relationships between the type of company and the risk of insolvency.

\subsection{Support Vector Machine (SVM)}

SVM is fundamentally a linear machine, which can be seen as a statistical tool that procedures the problem identical to Artificial Neural Networks (ANN). Structural Risk
Minimization (SRM) is used to relay wellbeing on unseen data.SVM is a similar implementation principle of Structural Risk minimization. While on one hand it has all the strengths of ANN, yet on the other hand it overcomes some of the basic lacunae as reported in the application of ANN (ASCE Task Committee, 2000a, b). In this paper, a brief discussion on the strengths of SVM over ANN is presented.

Now a day's researchers focused on SVM classifiers and its applications, also recently focused on regressions and time series predictions. Mukherjee et al. (1997) applied SVM for non-linear prediction of chaotic time series (the MackeyGlass time series, the Ikeda map and the Lorenz time series) and contrast the results with different similar techniques (ANN, polynomial, RBFs, local polynomial and rational).Finally in chaotic time series SVM gave good performance than the other techniques. Dibike (2000) rainfall lrunoff modeling says SVM is better than the ANN and genetic programming .Babovic et al. (2000) concluded that SVM produced systematically better results over 12 lead periods than ANN for water level predicting in the city of Venice. Liong \& Siva pragasam (2000) and Siva pragasam \& Liong (2000) concluded that in rainfall-runoff modeling and flood forecasting SVM gives better performance.

Text (and hypertext) categorization: Task: Based upon their content we convert natural text into predefined fixed number of categories .Sorting documents by topic, Email filtering, web searching etc.. A document can be allocated to more than one category, so this can be viewed as a series of binary classification problems, one for each category.

- Image classification

- Bioinformatics (Protein classification, Cancer classification)

- Hand-written character recognition

\subsection{Multilevel Clustering for large Databases}

For handling large data sets and multi-level data structures basic clustering techniques are failed [8]. These large data sets handle by using Self-Organizing map and vector machine with other clustering methods. More over in order to take into account multi-level structures, a statistical model is proposed, in which mixture of distributions may have mixing coefficients depending on higher-level variables. It is a two step process in step one, the SOM supply a considerable data reduction in step two provides statistical modeling. In data reduction we use different ascending and divisive clustering algorithms, statistical modeling provides both framework for model based clustering and a direct means to treat multilevel structures.

In this proposed work primarily focused on clustering it will be done by using SOM and SVM with ID3.The main characteristics of increasing hierarchical SOM are its hierarchical architecture, where the depth of the hierarchy is decided during an unsupervised training process. Each layer in the hierarchy contains of a number of Absolute SOM and SVM, which decide their size and arrangement of units also during the unsupervised training process. This model is well suited for applications which need hierarchical clustering of the input data, particularly for weather forecasting.

\section{SYSTEM STRUCTURE}

Data mining is mainly focused on clustering algorithms; Clustering can be considered the most important unsupervised 
learning problem. A cluster is therefore a collection of objects which are "similar" between them and are "dissimilar" to the objects belonging to other clusters. In the actual world actual prediction process has different approaches, here we shown one of the prediction process followed by India as shown in Fig 1. This process has dividing into two categories in the first category it predict the actual weather of the searched city, it contains future five days information with all the data like temperature, humidity, rainy, windy etc., in the second category it contains about the warning regarding the cyclones, thunder storms, heavy rains, etc.,[25]. The data Set of gathered from different sources of different climatic conditions of different places namely Indonesia, and other local places of India and processed through pre processing techniques.

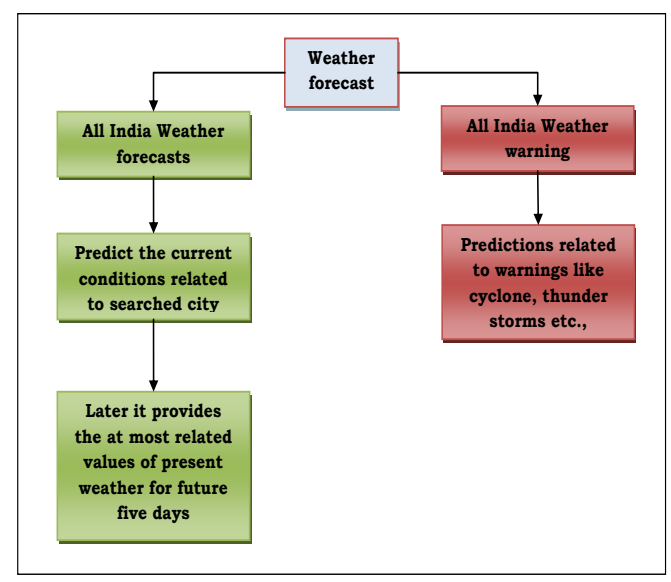

Fig 1 actual approach of the weather prediction

Our proposed work uses two level approaches for clustering. One is an unsupervised learning process i.e., Self-Organizing Map another one is an SVM algorithm with ID3 was shown in Fig 1. .More spectacularly, the learning features of ANN allow them to deal efficiently with noisy data partial, Inexact and possibly conflicting data. The use of ANN as data mining tools remains an area for further stydy. We use ANN as a feasible data mining tool and the capacity of Self-Organizing maps (SOM) and SVM as a helpful tool for the finding of statistical insights and models from large data sets, i.e. explorative data analysis. By using SOM and SVM with ID3, high-dimensional data can be projected to a lower dimension representation scheme (a two-dimensional map) that can be easily visualized and understood. More spectacularly, the transformation leads to an automatic clustering of the data, i.e. similar data items are stored in proximity thereby forming clusters. The Global Frame work Model, SVRK (Support Vector Regression Kernel Agent) for prediction in an efficient manner.

\subsection{SOM Algorithm}

Initially, the algorithm initializes weights of the map. By using three techniques we have to initialize weights of a map. The first technique is to use random values, entirely selfsufficient of the training data set. In this technique, map does not know about input data. It is a simple but rather poor way of initializing the map because it requires quite a number of additional training cycles until the map can be said to be at least roughly representative of the training data. The second approach is to use random samples from the input training data. The advantage is that the initial weight vectors already lie in the same space as the training data. When the training commences, the map is already in a state in which it represents at least a subset of the input data items. This obviously reduces the number of training iterations needed and hence lowers the computational cost. Nevertheless, the choice of input samples used for the initialization is random and the number of map nodes is very small compared to the number of training data items. The initial map is hence not likely to be truly representative of the given dataset. One may easily imagine a scenario where by pure coincidence a large number of outliers have been chosen which has little in common with the majority of data items. Similarly, the initial samples may not reflect the existence of outliers at all.

\subsection{SVM Algorithm}

It initializes the set with the near pair of points from opposite classes like the Direct SVM algorithm. As soon as the algorithm finds a violating point in the dataset it greedily adds into the candidate set. It may happen that addition of the violating point as a Support Vector may be prevented by other candidate Support Vectors already present in the set. We simply prune away all such points from the candidate set. We use the quadratic penalty formulation to ensure linear separability of the data points in the kernel space

First of all, we observe that finding the closest pair of points in kernel space requires $\mathrm{n} 2$ kernel computations where $\mathrm{n}$ represents the total number of data points. But, in worst case we use a distance preserving kernel like the exponential kernel the nearest neighbors in the feature space are the same as the nearest neighbors in the kernel space. Hence we need not perform any costly kernel evaluations for the initialization step. Given a set S which contains only Support Vectors, we wish to add another Support Vector c to S.

\subsection{ID3 Algorithm:}

ID3 builds a decision tree from a fixed set of examples. The resulting tree is used to classify future samples. The example has several attributes and belongs to a class (like yes or no). The leaf nodes of the decision tree contain the class name whereas a non-leaf node is a decision node. The decision node is an attribute test with each branch (to another decision tree) being a possible value of the attribute. ID3 uses information gain to help it decide which attribute goes into a decision node. The advantage of learning a decision tree is that a program, rather than a knowledge engineer, elicits knowledge from an expert. This basic structure is shown in Figure 3. The main ideas behind the ID3 algorithm are:

- In decision tree each non leaf node represents an attribute, and edge represents value of an attribute. A leaf node corresponds to the expected value of the output attribute when the input attributes are described by the path from the root node to that leaf node.

- In a "good" decision tree, each non leaf node is an attribute which is the most instructional about the output attribute amongst all the input attributes not yet considered in the path from the root node to that node. This is because we would like to forecast the output attribute using the smallest possible number of questions on average.

Training Data and Set: ID3 is a supervised learner. It needs to have training data sets to make decisions. ID3 is a supervised learning algorithm it needs training data set for processing. The training gives attributes and their values. ID3 doesn't deal with continuous, numeric data which means we have to discretize them. Dataset, there are five categorical attributes outlook, temperature, windy, humidity, and rainy. Now we are find one system for forecasting weather. The 
attributes such as outlook, temperature, humidity, windy, rainy which can values are shown in Table 1 and 2 .

Table 1: Data attributes

\begin{tabular}{|l|l|}
\hline & Values \\
\hline outlook & sunny \\
\hline temperature & 85 \\
\hline humidity & 85 \\
\hline windy crop & FALSE \\
\hline rainy & NO \\
\hline
\end{tabular}

Table 2: Actual data

\begin{tabular}{|l|l|l|l|l|}
\hline Weather & Temp & Humidity & Wind & Crop \\
\hline sunny & 85 & 85 & false & no \\
\hline sunny & 80 & 90 & true & no \\
\hline overcast & 83 & 86 & false & no \\
\hline rainy & 70 & 96 & false & yes \\
\hline rainy & 68 & 80 & false & yes \\
\hline rainy & 65 & 70 & true & no \\
\hline overcast & 64 & 65 & true & yes \\
\hline
\end{tabular}

\subsection{Entropy}

A decision tree is built top-down from a root node and involves partitioning the data into subsets that contain instances with similar values (homogenous). ID3 algorithm uses entropy to calculate the homogeneity of a sample. If the sample is completely homogeneous the entropy is zero and if the sample is an equally divided it has entropy of one.

$E(S)=-(p+) * \log 2(p+)-(p-) *$ $\log 2(p-)$

"S" represents the set and " $p+$ " are the number of elements in the set "S" with positive values and "p-" is the number of elements with negative values. The purpose of ID3 algorithm is to classify data using decision trees, such that the resulting leaf nodes are all homogenous with zero entropy.

\subsection{Gain}

The information gain is based on the decrease in entropy after a dataset is split on an attribute. Constructing a decision tree is all about finding attribute that returns the highest information gain (i.e., the most homogeneous branches). It is computed as

\section{$\operatorname{Gain}(S, A)=\operatorname{Entropy}(S)-S\left(\left(\left|S_{V} / /\right| S \mid\right){ }^{*} \operatorname{Entropy}\left(S_{V}\right)\right)$} (2)

Where, ' $\mathrm{S}$ ' is the set and ' $\mathrm{A}$ ' is the attribute. ' $\mathrm{SV}$ ' is the subset of ' $S$ ' where attribute ' $A$ ' has value ' $v$ '. ' $|S|$ ' is the number of elements in set ' $\mathrm{S}$ ' and ' $\mathrm{Sv} \mid$ ' is the number of elements in subset 'Sv'. ID3 chooses the attribute with the highest gain to create nodes in the decision tree. If the resulting subsets do not have entropy zero or equal to zero then it chooses one of the remaining attribute to create further nodes until all the subsets are homogenous.

\section{RESULTS}

In this paper, SOM and SVM give procedure for automatically learning a recognition model and also showed their efficiency. Gesture recognition has many potential applications. This method is applied to high dimensional data of rain fall data. In our approach we consider the SOM and SVM with ID3 algorithms, here we consider the different predictions for rainy or not which are shown in Fig 5. In this implementation we consider attributes which are shown in table 3 and 4. For conducting experiments we consider rain fall data, Input Data set is numerical data values having variables as rainfall at that $\mathrm{hr}$, temperature, humidity, dew point, sea level pressure, Predicting rainfall occurs at case, yes case.. if yes ,..is it high or moderate, low level ..in mm values. These are shown in Table 5 and draw the graph shown in Fig 2 for these results, which shows that the prediction entirely depending on temperature and breezy percentage.

Table 3: data

\begin{tabular}{|l|}
\hline if temperature $=85$ then Prediction $=$ no \\
\hline if temperature $=80$ then Prediction $=$ no \\
\hline if temperature $=83$ then Prediction $=y e s$ \\
\hline if temperature $=70$ then Prediction $=y e s$ \\
\hline if temperature $=68$ then Prediction $=\mathrm{yes}$ \\
\hline if temperature $=65$ then Prediction $=$ no \\
\hline if temperature $=64$ then Prediction $=y e s$ \\
\hline if temperature $=81$ then Prediction $=\mathrm{yes}$ \\
\hline if temperature $=71$ then Prediction $=\mathrm{no}$ \\
\hline
\end{tabular}

Table 4: Results

\begin{tabular}{|c|c|}
\hline Temperature & Prediction \\
\hline 85 & NO \\
\hline 80 & NO \\
\hline 83 & YES \\
\hline 70 & YES \\
\hline 72 & NO \\
\hline 69 & YES \\
\hline
\end{tabular}

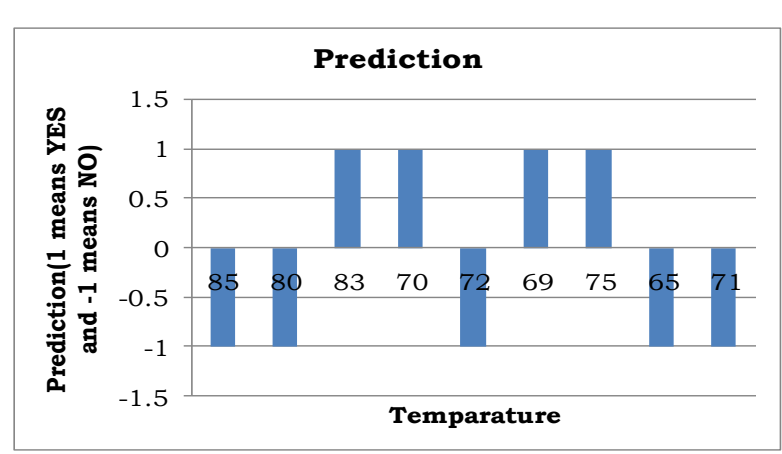

Fig 2. Prediction results

Table 5: Actual data set

\begin{tabular}{|c|c|c|c|c|c|}
\hline Day & Weather & Temp & Humidity & Wind & Crop \\
\hline 1. & Sunny & 85 & 85 & FALSE & NO \\
\hline 2. & Sunny & 80 & 90 & TRUE & NO \\
\hline
\end{tabular}




\begin{tabular}{|c|c|c|c|c|c|}
\hline 3. & Cloudy & 83 & 78 & FALSE & YES \\
\hline 4. & Rainy & 70 & 96 & FALSE & YES \\
\hline 5. & Rainy & 68 & 80 & FALSE & YES \\
\hline 6. & Rainy & 65 & 70 & TRUE & NO \\
\hline 7. & Cloudy & 64 & 65 & TRUE & YES \\
\hline 8. & Sunny & 72 & 95 & FALSE & NO \\
\hline 9. & Sunny & 69 & 70 & FALSE & YES \\
\hline 10. & Rainy & 75 & 80 & FALSE & YES \\
\hline 11. & Sunny & 75 & 70 & TRUE & YES \\
\hline 12. & Cloudy & 72 & 90 & TRUE & YES \\
\hline 13. & Cloudy & 81 & 75 & FALSE & YES \\
\hline 14. & Rainy & 71 & 85 & TRUE & NO \\
\hline
\end{tabular}

After applying our proposed approach (i.e. SOM, SVM with ID3) Table 4 as input data set we get the actual proposed prediction. While comparing the actual data set Table 5 and prediction values Table 6 we clearly observe that there is almost $82 \%$ accuracy. Actually this is predicted based on the temperature and humidity and wind strongest. Because of this accuracy these proposed approach can out performs. The studies and their implementation conducted here conclude that the decision tree learning algorithm ID3 works well on any classification problems having dataset with the discrete values. Related to the research work it concludes that thus the classification tree built using ID3 algorithm is performs well. It tells if the weather was amenable to crop or not. The classified datasets, having soft margins of the framed design is modularized to have very separable data in order to analyze the output of result analysis.

Table 6: Prediction Dataset

\begin{tabular}{|l|l|l|l|l|l|}
\hline Day & Weather & Temp & Humdity & Wind & play \\
\hline 1 & Sunny & 85 & 85 & False & Yes \\
\hline 2 & Sunny & 80 & 90 & True & Yes \\
\hline 3 & Cloudy & 83 & 78 & False & Yes \\
\hline 4 & Rainy & 70 & 96 & False & Yes \\
\hline 5 & Rainy & 68 & 80 & False & Yes \\
\hline 6 & Rainy & 65 & 70 & True & No \\
\hline 7 & cloudy & 64 & 65 & True & Yes \\
\hline 8 & Sunny & 72 & 95 & False & Yes \\
\hline 9 & Sunny & 69 & 70 & False & Yes \\
\hline 10 & Rainy & 75 & 80 & False & Yes \\
\hline 11 & Sunny & 75 & 70 & True & Yes \\
\hline 12 & Cloudy & 72 & 90 & True & Yes \\
\hline 13 & Cloudy & 81 & 75 & False & Yes \\
\hline
\end{tabular}

\begin{tabular}{|l|l|l|l|l|l|}
\hline 14 & Raimy & 71 & 85 & True & No \\
\hline 15 & Sunny & 80 & 90 & True & Yes \\
\hline 16 & Rainy & 70 & 96 & False & Yes \\
\hline 17 & Rainy & 68 & 80 & False & Yes \\
\hline 18 & Rainy & 65 & 70 & True & No \\
\hline 19 & Sunny & 72 & 95 & False & Yes \\
\hline 20 & Sunny & 69 & 70 & False & yes \\
\hline
\end{tabular}

\section{CONCLUSION}

For prediction and visualization of large collection of data sets SOM and SVM are the best machine learning techniques. In this proposed work we implement two level architecture for visualization and prediction. First, the data set is divided into small set of vectors and then using clustering algorithms these vectors will be divided into groups. Because, the main aim of hierarchical algorithms is to reduce computational cost for each cluster. Second, it gives rough visualization for each cluster. Thus, it finally has advantages over previous approaches with prediction, cost, reliability and computation wise. The soft computing procedures can also able to give better visualization and predictions.

\section{REFERENCES}

[1] Bhowmik RS, Durai V (2010) Application of multi model ensemble techniques for real time district level rainfall forecasts in short range time scale over Indian region. Meteorol Atmos Phys 106:19-35.

[2] Březková L, Šálek M, Soukalová EC (2010) Predictability of flood events in view of current meteorology and hydrology in the conditions of the Czech Republic. Soil Water Res 2(4):156-168

[3] Cavazos T, Turrent C, Lettenmaier DP (2008) Extreme precipitation trends associated with tropical cyclones in the core of the North American monsoon. Geophys Res Lett 35:L21703

[4] Daoud BA, Sauquet E, Lang M, Bontron G, Obled C (2011) Precipitation forecasting through an analog sorting technique: a comparative study. Adv Geosci 29:103-107

[5] Dodla VBR, Ratna SB (2010) Mesoscale characteristics and prediction of an unusual extreme heavy precipitation event over India using a high resolution mesoscale model. Atmos Res 95:255-269. doi:10.1016/j.atmosres.2009.10.004.

[6] Hong S-Y, Lee J-W (2009) Assessment of the WRF model in reproducing a flash-flood heavy rainfall event over Korea. Atmos Res 93:818-831. doi:10.1016/j.atmosres.2009.03.015

[7] Khaladkar RM, Narkhedkar SG, Mahajan PN (2007) Performance of NCMRWF models in predicting high rainfall spells during SW monsoon season-a study for some cases in July 2004. Research Report No. RR-116

[8] Narkhedkar SG, Sinha SK, Mitra A (2008) Mesoscale objective analysis of daily rainfall with satellite and 
conventional data over Indian summer monsoon region. Geofizika 25:159-177

[9] Rajendra KJ, Bhan SC, Kalsi SR (2006) Observational/forecasting aspects of the meteorological event that caused a record highest rainfall in Mumbai. Current Science Association, Bangalore, INDE; 19

[10] Tymvios F, Savvidou K, Michaelides SC (2010) Association of geopotential height patterns with heavy rainfall events in Cyprus. Adv Geosci 23:73-78 CrossRef

[11] Ronan Billon, Alexis Nedelec and Jacques Tisseau, "Gesture recognition in flow based on PCA and using multiagent system", Proc. of Advances on Computer Entertainment 2008,pp.139-146, 2008.

[12] Juha Vesanto and Esa Alhoniemi,"Clustering of the Self -Organizing Map", IEEE transactions on Neural Networks vol.11, No.3 , may 2000

[13] A.K. Jain, Michigan State University, M.N. Murthy, Indian Institute of Science and P.J. Flynn The Ohio State University, "Data Clustering, a Review" ACM Computing Surveys, Vol. 31, No. 3, September 1999

[14] A.K. Jain, Robert P.W.Duin and J.Mao, Senior Members, IEEE. "Statistical Pattern Recognition, a Review" Teuvo kohonen , "The Self Organizing Map", proceedings of the IEEE vol.78, No. 9, September 1990

[15] Jouko Lampinen and Ekki Oja, Lappeenranta University of Technology,Departament of Information Technology "Clustering Properties of Hierarchical Self-Organizing maps"

[16] Michael Dittenbach,Dieter Merkl, Andreas Rauber "The Growing Hierarchical Self-Organizing Map" proceedings of Int'l Joint Conference on Neural Networks, como, Italy VI-15-VI-19 July 2000
[17] Yves Lechevallier and Antonio Ciampi "Multilevel Clustering for large Databases" Department of Epidemiology and Biostatistics, McGill University ,Canada

[18] Timo Honkela "Self Organizing Maps in Natural Language Processing" Helsinki University of Technology

[19] Jost Schatzmann "Using Self-Organizing Maps to Visualize Clusters and Trends in Multidimensional Datasets" Supervisor: Dr Moustafa Ghanem Imperial College London 180 Queen's Gate London, UK 19 June 2003 , London .

[20] Juha vesanto "SOM Based Data Visualization Methods", Laboratory of Computer and Information Science, Helenski University of Technology, Finland.

[21] Jason Ong "Data Mining Using Self-Organizing Kohonen maps: A technique for Effective Data Clustering and Visualization" School of Computer Sciences University of Malasia

[22] George Karpis, Eui-Hong(Sam)Han, Vipin Kumar "Chameleon: A Hierarchical Clustering Algorithm Using Dynamic Modeling"

[23] Kaski, S., "Data exploration using self-organizing maps" Acta Polytechnica Scandinavica, Mathematics, Computing and Management in Engineering Series No. 82, Espoo 1997, 57 pp. Published by the Finnish Academy of Technology.

[24] Subimal Ghosh, Munir Ahmad Nayak, Prediction of extreme rainfall event using weather pattern recognition and support vector machine classifier, Theoretical and Applied ClimatologyNovember 2013, Volume 114, Issue 3-4, pp 583-603, 06 Mar 2013. 